\title{
Metallomics
}

Cite this: Metallomics, 2013, 5, 318

Received 7th September 2012, Accepted 30th October 2012 DOI: $10.1039 / \mathrm{c} 2 \mathrm{mt} 20180 \mathrm{a}$ www.rsc.org/metallomics

\section{Cold-adapted arsenite oxidase from a psychrotolerant Polaromonas species $\dagger$}

\author{
Thomas H. Osborne, ${ }^{a}$ Matthew D. Heath, ${ }^{a}$ Andrew C. R. Martin, ${ }^{a}$ \\ Jaroslaw A. Pankowski, ${ }^{a}$ Karen A. Hudson-Edwards ${ }^{b}$ and Joanne M. Santini*a
}

\begin{abstract}
Polaromonas sp. str. GM1 is an aerobic, psychrotolerant, heterotrophic member of the Betaproteobacteria and is the only isolate capable of oxidising arsenite at temperatures below $10{ }^{\circ} \mathrm{C}$. Sequencing of the aio gene cluster in GM1 revealed the presence of the $a i o B$ and aio $A$ genes, which encode the arsenite oxidase but the regulatory genes typically found upstream of $a i o B$ in other members of the Proteobacteria were absent. The GM1 Aio was purified to homogeneity and was found to be a heterodimer. The enzyme contained $\mathrm{Mo}$ and $\mathrm{Fe}$ as cofactors and had, using the artificial electron acceptor 2,6-dichlorophenolindophenol, a $K_{\mathrm{m}}$ for arsenite of $111.70 \pm 0.88 \mu \mathrm{M}$ and a $V_{\max }$ of $12.16 \pm 0.30 \mathrm{U} \mathrm{mg}^{-1}$, which is the highest reported specific activity for any known Aio. The temperature-activity profiles of the arsenite oxidases from GM1 and the mesophilic betaproteobacterium Alcaligenes faecalis were compared and showed that the GM1 Aio was more active at low temperatures than that of $A$. faecalis. A homology model of the GM1 Aio was made using the X-ray crystal structure of the Aio from $A$. faecalis as the template. Structural changes that account for cold adaptation were identified and it was found that these resulted in increased enzyme flexibility and a reduction in the hydrophobicity of the core.
\end{abstract}

\section{Introduction}

Arsenic is a group 5 metalloid and is renowned for its toxicity. ${ }^{1}$ The soluble inorganic forms of arsenic, arsenite (+3) and arsenate $(+5)$, are widespread in terrestrial and aquatic environments, ${ }^{2}$ where arsenite is the more toxic and mobile species. ${ }^{3}$ The consumption of naturally occurring inorganic arsenic in drinking water has caused poisoning of human populations across regions of West Bengal, Bangladesh, Chile, Taiwan and Vietnam, while anthropogenic activity (principally through the mining of valuable metals) has resulted in arsenic contamination of environments in Canada, USA, Argentina and Australia. ${ }^{4,5}$ Despite its toxicity, certain microbes are capable of gaining energy by oxidising arsenite to arsenate, ${ }^{6}$ and this process contributes to the global biogeochemical cycling of arsenic. ${ }^{7}$ Since the first discovery of bacterial arsenite oxidation by Green in $1918,{ }^{8}$ over 50 strains of arsenite-oxidising bacteria have been isolated. The arsenite-oxidising bacteria are phylogenetically diverse and have been isolated from different environments (reviewed by Osborne and Santini). ${ }^{9}$

\footnotetext{
${ }^{a}$ Institute of Structural and Molecular Biology, University College London, Gower Street, London,WC1E 6BT, UK. E-mail: j.santini@ucl.ac.uk

${ }^{b}$ Department of Earth and Planetary Sciences, Birkbeck, University of London, Malet Street, London, WC1E 7HX, UK

† Electronic supplementary information (ESI) available. See DOI: 10.1039/c2mt20180a
}

Aerobic oxidation of arsenite is catalysed by arsenite oxidase (Aio) (see Lett et al. for recent changes to the nomenclature for the arsenite oxidase). ${ }^{10}$ Aio is a member of the DMSO reductase family of molybdoenzymes which is a family of redox enzymes that function in a diverse range of prokaryotic electron transport chains. ${ }^{11}$ The enzyme is a heterodimer that consists of a large $\alpha$-subunit, AioA and a small $\beta$-subunit, AioB. The X-ray crystal structure of the Aio from the mesophilic, heterotrophic betaproteobacterium Alcaligenes faecalis has been resolved. ${ }^{12}$ The structure shows that AioA contains a $3 \mathrm{Fe}-4 \mathrm{~S}$ cluster and a bis-molybdopterin guanine dinucleotide (bis-MGD) cofactor at the active site, while AioB contains a $2 \mathrm{Fe}-2 \mathrm{~S}$ Rieske cluster. The Aio is exported to the periplasm by the Twin-arginine translocation (Tat) pathway conferred by an N-terminal signal sequence on AioB. The Aio that have been characterised to date have all been purified from mesophilic arsenite oxidisers (reviewed by Heath et al.). ${ }^{13}$

The aio operon structure always includes the aio $A$ and aioB genes, with aioB located upstream of aioA. In some arsenite oxidisers of the Alpha- and Betaproteobacteria a cytochrome $c$-encoding gene $(c y t C)$ is located downstream of aio $A^{9}$ and this cytochrome has been shown to serve as the electron acceptor to the Aio. ${ }^{14,15}$ Often found upstream of aioB are three genes (aioXSR) whose products are involved in the regulation of arsenite oxidase gene expression. Aios is a sensor histidine kinase and AioR a response regulator. ${ }^{16}$ Together with a third 
gene product, AioX, which has been shown to bind arsenite, ${ }^{17}$ they are thought to regulate transcription of the aioBA operon. Variation is seen in the other genes found in the aio cluster. Of the isolated arsenite oxidisers, only Polaromonas sp. str. GM1, an aerobic, heterotrophic, betaproteobacterium, has been shown to oxidise arsenite at temperatures below $10{ }^{\circ} \mathrm{C} .{ }^{18} \mathrm{GM} 1$ is also unusual in that aio gene expression is constitutive and it oxidises arsenite in the early log-phase of growth whereas most heterotrophic arsenite oxidisers oxidise arsenite in the late-log or stationary phase using arsenite as the secondary energy source. GM1's Aio is constitutively expressed which may be explained by the environment in which GM1 was isolated, an arseniccontaminated biofilm that contained about $10 \mathrm{mM}$ arsenic. ${ }^{18}$ In this paper we present a study of arsenite oxidation in GM1 and its cold-adapted arsenite oxidase. Understanding the basis of the enzyme's cold-adaptation could result after some improvement in its use as a broad-temperature range biosensor.

\section{Results and discussion}

GM1 can oxidise arsenite at $0{ }^{\circ} \mathrm{C}$ (data not shown) to $20{ }^{\circ} \mathrm{C}$ and does so in the early-log phase of growth, ${ }^{18}$ which is unusual for heterotrophic arsenite oxidisers. The Aio of GM1 is functional over a broad temperature range and its expression was found to be constitutively expressed and up-regulated in the late-log phase of growth. ${ }^{18}$ The data presented here contribute to a better understanding of arsenite oxidation and how the GM1 Aio is able to function at low temperatures.

\section{Identification of the aio gene cluster}

The aioA gene of GM1 was mutated by targeted gene disruption generating GM1-A. This mutant was unable to oxidise arsenite and did not exhibit detectable Aio activity (using DCPIP as the electron acceptor) in total cell extracts (data not shown), confirming that aioA confers an arsenite-oxidising phenotype in GM1. Mutagenesis of aioA facilitated the cloning of a $5.3 \mathrm{~kb}$ fragment which was sequenced. The 5307 bp sequence contains four complete and one partial open reading frame: this includes a putative phage integrase (partial sequence), the arsenite oxidase small subunit (aioB), the arsenite oxidase large subunit (aioA), a putative $c$-type cytochrome (cytC) and a nitroreductase (Fig. 1). These genes are transcribed in the same direction. No consensus sequence for a promoter was identified. Genes involved in the regulation of arsenite oxidation (i.e. aioXSR) were not identified but this is not surprising given that arsenite oxidation in GM1 is constitutively expressed whereas in bacteria that contain aioXSR the expression of the aioBA operon is induced with arsenite. ${ }^{15,20,21}$

The partial open reading frame (1059 nt of a possible $1428 \mathrm{nt}$ encoding 353/476 amino acids) upstream of aioB was found to share homology with putative phage integrases from various Betaproteobacteria such as Oxalobacteraceae sp. str. IMCC9480 (59\% identity over 172 amino acids) and Cupriavidus (also known as Ralstonia) metallidurans (40\% identity over 317 amino acids). The GM1 integrase sequence however, did not contain the conserved C-terminal catalytic domain present in the sequences of the closely related phage integrases, indicating that it is unlikely to be functional. The $a i o B$ gene is found downstream of the partial integrase sequence and encodes an apoprotein of 179 amino acids (18561 Da). The protein shares closest sequence identity with the AioB homologues in the Betaproteobacteria such as Acidovorax sp. str. NO-1 (73\% identity), A. faecalis (57\% identity) and Herminiimonas arsenicoxydans (61\% identity) compared with the alphaproteobacterial sequence from NT-26 (40\% identity). The sequence contains a conserved motif involved in binding the Rieske $2 \mathrm{Fe}-2 \mathrm{~S}$ cluster and in the case of $A$. faecalis (not in NT-26) conserved residues involved in disulphide bond formation, Cys-X-His-X-Gly-Cys*$\mathrm{X}_{17}$-Cys-X-Cys ${ }^{*}$-His (cluster ligands are in italics; the asterisked residues form a disulphide bond which bridges over the cluster, connecting the two cluster-binding loops). In common with all other AioB sequences the GM1 sequence contains a Tat signal sequence (1-36 amino acids) with the conserved RRXFLK motif $^{22}$ (RRFFLK in GM1 AioB) which directs the transport of the fully folded AioBA complex to the periplasm via the Tat pathway. A predicted signal cleavage site is present between amino acids 36 and 37 (i.e. ADA-AT) resulting in a processed protein of $14733 \mathrm{Da}$. Downstream of aioB is aioA and it encodes a protein of 827 amino acid (91690 Da unprocessed and 91559 Da without N-terminal methionine). The GM1 AioA shares the highest sequence identity with that from Acidovorax sp. str. NO1 (80\% identity), A. faecalis (70\% identity) and Herminiimonas arsenicoxydans (69\% identity) compared with that from NT-26 (47\% identity). The conserved motif, Cys- $\mathrm{X}_{2}$-Cys- $\mathrm{X}_{3}$-Cys-X-Ser70 involved in binding the $3 \mathrm{Fe}-4 \mathrm{~S}$ cluster, is conserved, as are the amino acids implicated in binding arsenite (i.e. His197, Glu205, Arg421 and His425). Downstream of aioB is $c y t C$, which encodes a putative $c$-type cytochrome of 104 amino acids (10 884 Da unprocessed) and contains a single $\mathrm{CX}_{2} \mathrm{CH}$ heme-binding motif suggesting the presence of a single heme $c$. The protein also

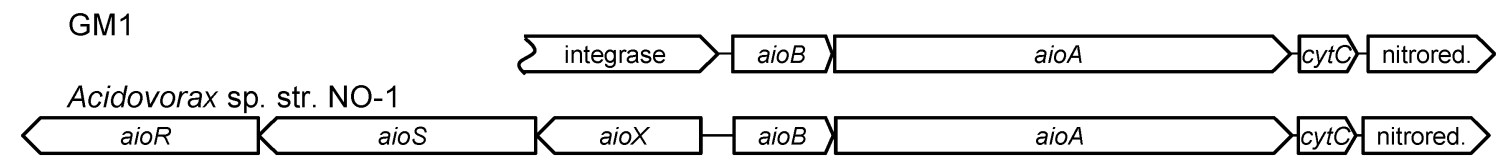

$500 \mathrm{bp}$

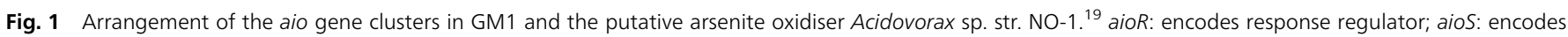

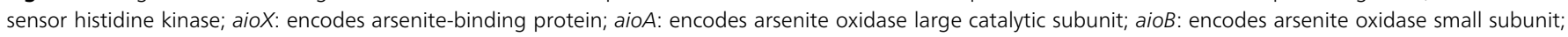
cytC: encodes putative c-type cytochrome. Acidovorax sp. str. NO-1 accession number: HM357240.1. 
contains a general secretory pathway (Sec) signal sequence for its transport to the periplasm, with a predicted cleavage site between amino acids 22 and 23 (i.e. SFA-NL) resulting in a processed protein of $8503 \mathrm{Da}$. The putative cytochrome may act as a physiological electron acceptor to the GM1 Aio as was observed for the $c_{552}$ cytochrome encoded by the cytC gene in the aio cluster of NT-26. ${ }^{15}$ The putative GM1 cytochrome and nitroreductase did not show significant similarity to cytochrome and nitroreductase encoding genes in other aio gene clusters.

\section{Purification and characterisation of the arsenite oxidase}

The Aio from GM1 was purified from the periplasm by a two-step ammonium sulphate precipitation (50\% and 75\%) followed by cation-exchange and size-exclusion chromatography. The Aio was enriched 32.7-fold (Table 1). The purified Aio consisted of two heterologous subunits of $88 \mathrm{kDa}$ (AioA) and $15 \mathrm{kDa}$ (AioB) (Fig. 2), and had a native molecular mass of $105.5 \mathrm{kDa}$ (determined by gel filtration chromatography) indicating that the enzyme is a heterodimer $\left(\alpha_{1} \beta_{1}\right)$. The oligomeric state of the GM1 Aio is like that of A. faecalis, ${ }^{23}$ Arthrobacter sp. str. $15 b^{24}$ and Ralstonia sp. str. $22^{14}$ but varies from that of NT-26 ${ }^{25}$ and Hydrogenophaga sp. str. NT-14 ${ }^{26}$ which form oligomers of $\alpha_{2} \beta_{2}$ and $\alpha_{3} \beta_{3}$, respectively.

Purified GM1 Aio was analysed for metal cofactors using ICP-MS and was found to contain Mo (1.2 mol mol ${ }^{-1}$ enzyme) and Fe (6 mol mol ${ }^{-1}$ enzyme), indicating that like the mesophilic enzymes, the GM1 Aio contains Mo at the active site and two Fe-S clusters, which was also confirmed by sequence analyses (see above). The enzyme had a $K_{\mathrm{m}}$ for arsenite of $111.70 \pm 0.88 \mu \mathrm{M}$ and a $V_{\max }$ of $12.16 \pm 0.30 \mu \mathrm{mol}$ arsenite oxidised $\min \mathrm{mg}^{-1}$, corresponding to a $K_{\text {cat }}$ of $21.38 \pm 0.52 \mathrm{~s}^{-1}$ (based on a native molecular mass of $105.5 \mathrm{kDa}$ ). All three of these values are the highest observed for any Aio purified to date, when DCPIP was used as the electron acceptor. The higher $K_{\mathrm{m}}$ value shows that the GM1 Aio has a lower affinity for arsenite than the mesophilic enzymes, while the high $V_{\max }$ value is at least double that of the most active mesophilic Aio (i.e. that of NT-14). ${ }^{14,25,26}$

A variety of $c$-type cytochromes and the copper-containing protein azurin can serve as electron acceptors to the Aio. NT-26 cytochrome $c_{552}$ (electron acceptor to the NT-26 Aio), ${ }^{15}$ commercially purchased horse heart cytochrome $c$ and Pseudomonas aeruginosa azurin, were tested for their ability to accept electrons from the GM1 Aio. Cytochrome $c_{552}$ and horse-heart cytochrome $c$ were not reduced by the GM1 Aio in the presence of arsenite (ESI, $\uparrow$ Fig. S1 and S2). The oxidised $P$. aeruginos $a$ azurin exhibited a characteristic

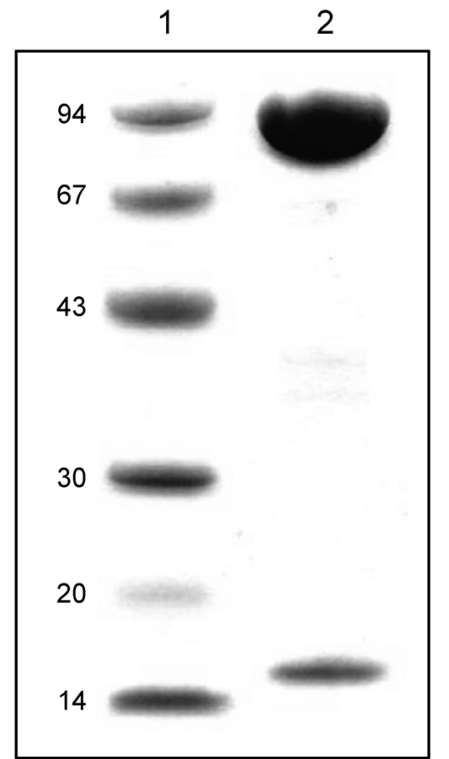

Fig. 2 SDS-polyacrylamide gel of purified GM1 Aio stained with Coomassie Blue R350. 1: low molecular weight standards (size in $\mathrm{kDa}$ ); 2: purified GM1 Aio.

absorption band at $625 \mathrm{~nm}$ which disappeared upon its reduction in the presence of the GM1 Aio and arsenite (Fig. 3). Azurin was not reduced by either the Aio or arsenite alone (ESI, $\uparrow$ Fig. S3). The Aio of A. faecalis and Ralstonia sp. str. 22 can also use azurin as the electron acceptor but like the GM1 Aio are unable to use horse heart cytochrome $c$ and the NT-26 $c_{552}{ }^{14,23}$ whereas the NT-26 Aio which can use the $c_{552}$ and horse heart cytochrome $c$ cannot use azurin. ${ }^{15}$ The reason why the enzymes react differently with the various electron acceptors is presently unknown, but it appears to be unrelated to the redox potentials of the Rieske cluster. ${ }^{14,15,27}$

\section{Cold adaptation of GM1 Aio}

GM1 is a psychrotolerant arsenite oxidiser with a growth range between $0-20{ }^{\circ} \mathrm{C}$. We therefore tested the effect of temperature on Aio activity making comparisons with the mesophilic enzyme from the only organism with a solved X-ray crystal structure A. faecalis. ${ }^{12}$ The enzyme from A. faecalis was purified after successful heterologous expression in E. coli. GM1 Aio had a maximum observed activity at $45{ }^{\circ} \mathrm{C}\left(13.8 \mathrm{U} \mathrm{mg}^{-1}\right)$, which was reduced to $8.0 \mathrm{U} \mathrm{mg}^{-1}$ at $60{ }^{\circ} \mathrm{C}$ and $9.2 \mathrm{U} \mathrm{mg}^{-1}$ at $10{ }^{\circ} \mathrm{C}$ corresponding to reductions of $42 \%$ and $33 \%$ of maximum observed activity, respectively (Fig. 4). In contrast, the Aio from

Table 1 Purification of the GM1 Aio

\begin{tabular}{|c|c|c|c|c|}
\hline Purification step & Total protein (mg) & Total activity (U) & Specific activity $\left(\mathrm{U} \mathrm{mg}^{-1}\right)$ & Purification (-fold) \\
\hline Total cell extract & 74.4 & 26.83 & 0.36 & 1 \\
\hline Periplasm & 12.9 & 12.71 & 1.02 & 2.8 \\
\hline Desalting & 6.74 & 12.94 & 1.92 & 5.3 \\
\hline Cation exchange & 0.51 & 3.73 & 7.32 & 20.3 \\
\hline Gel filtration & 0.14 & 1.65 & 11.79 & 32.7 \\
\hline
\end{tabular}

The GM1 Aio was purified from the periplasm after the preparation of spheroplasts. The periplasm was fractionated by ammonium sulphate precipitation and the Aio-containing fraction desalted and purified by cation exchange and gel filtration chromatography. 


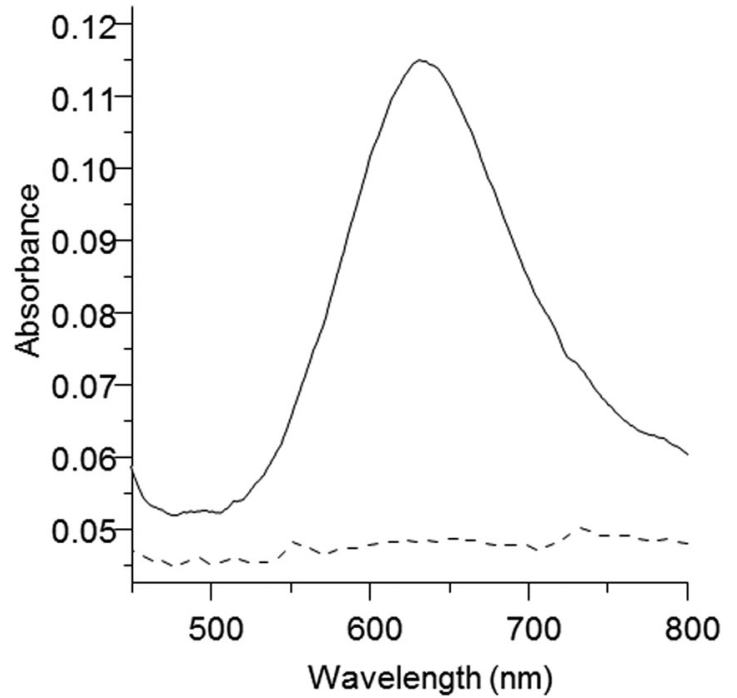

Fig. 3 Oxidised and reduced spectra of $P$. aeruginosa azurin. Oxidised azurin (solid line) was reduced (dashed line) in the presence of the GM1 Aio and arsenite.

A. faecalis had a maximum observed activity at $65{ }^{\circ} \mathrm{C}\left(8.1 \mathrm{U} \mathrm{mg}^{-1}\right)$, which was reduced to $7.3 \mathrm{U} \mathrm{mg}^{-1}$ at $75{ }^{\circ} \mathrm{C}$ and $1.2 \mathrm{U} \mathrm{mg}^{-1}$ at $10^{\circ} \mathrm{C}$, corresponding to reductions of $10 \%$ and $75 \%$ of maximum observed activity, respectively. As can be seen in Fig. 4, the GM1 Aio was active at lower temperatures (Fig. 4). The fact that the GM1 Aio had a lower optimum temperature $\left(45^{\circ} \mathrm{C}\right)$ than the $A$. faecalis enzyme $\left(65^{\circ} \mathrm{C}\right)$ and was also more active at lower temperatures is a common trait of cold-adapted enzymes. ${ }^{28}$

The properties that make enzymes cold-adapted are well known. ${ }^{28,29}$ The rate of an enzyme-catalysed reaction will change exponentially with a change in temperature hence temperature is in the exponent of the Arrhenius equation. Temperature is a function of activation energy and so a decrease in activation energy will make a reaction less dependent on temperature. In general, cold-adapted enzymes have lower

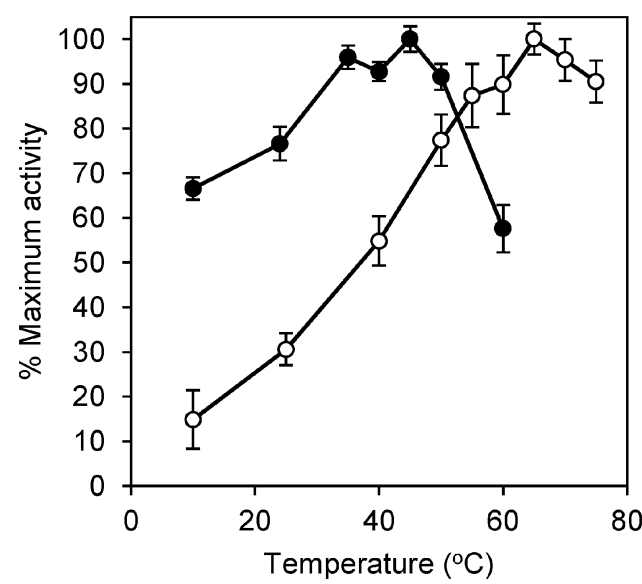

Fig. 4 Temperature-activity profiles of the GM1 and A. faecalis arsenite oxidases. Percentage of maximum activity is plotted as a function of temperature of GM1 (-) and A. faecalis ( $\bigcirc)$ Aio. Data points and error bars represent mean and standard deviation of at least three assays, respectively. activation energies than their mesophilic homologues increasing their activity at lower temperatures, which results from reduced enthalpy-requiring conformational changes needed to achieve the enzyme-substrate transition complex. The reduction in enthalpyrequiring changes often manifests itself as an increase in overall enzyme flexibility or localised flexibility around the active site, however, the increase in flexibility also decreases the protein's stability. ${ }^{28,29}$ The increase in flexibility to achieve increased reaction rates means that often cold-adapted enzymes also have decreased affinity for the substrate (i.e. higher $\left.K_{\mathrm{m}}\right)^{30,31}$ compared to their mesophilic homologues. An increased reaction rate and a decreased affinity for arsenite are observed for the GM1 Aio when compared to the mesophilic homologues suggesting that the former has increased flexibility.

\section{Homology modelling of GM1 Aio}

To investigate the molecular basis of cold-adaptation in the GM1 Aio, a model was built of the enzyme using the A. faecalis Aio crystal structure as a template. The model shows that the majority of amino acid differences between the two enzymes occur on the surface of the enzyme and that the entry channel to the active site is well conserved (Fig. 5). Destabilising effects on the structure, caused by amino acid differences between the GM1 and A. faecalis Aio were predicted using the SAAPdap pipeline of SAAPdb. ${ }^{32} 44$ amino acid differences (40 in AioA and 4 in AioB) were predicted to have destabilising structural effects on GM1 Aio, and are summarised in Table 2. It should be noted that as the analysis treats each amino acid difference as an individual mutation, many structural changes may be compensated by other changes. Increased flexibility/decreased stability of cold-adapted enzymes can be caused by several structural changes, including a decrease in stabilising bonds (H-bonds, van der Waals interactions and salt bridges), an increase in the surface hydrophobicity and a decrease in the core hydrophobicity. The SAAPdap analysis shows that a decrease in H-bonds and insertion

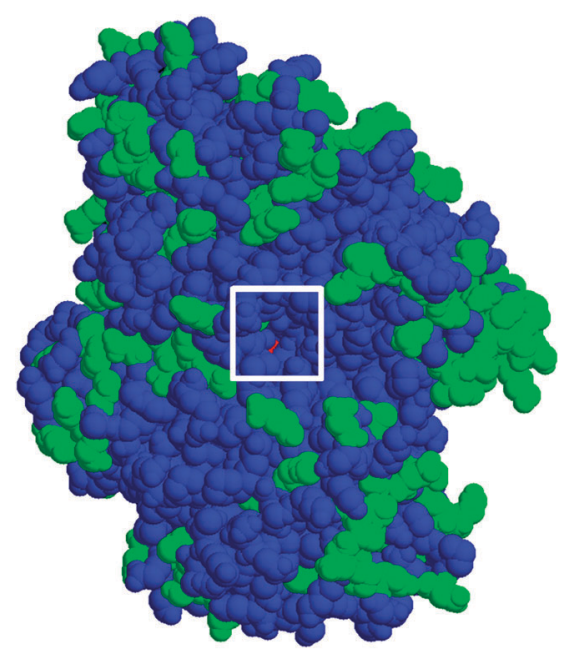

Fig. 5 Space-filling homology model of GM1 Aio. The channel to the active site is boxed. Conserved residues between the GM1 and $A$. faecalis structure are blue; different residues are green. 
Table 2 Destabilising structural effects of amino acid differences between GM1 and $A$. faecalis Aio identified by SAAPdap analysis

\begin{tabular}{lc}
\hline Structural effect & No. of amino acid differences \\
\hline HBond $^{a}$ & 22 \\
CorePhilic $^{b}$ & 10 \\
SurfacePhobic $^{c}$ & 6 \\
Buriedcharge $^{d}$ & 6 \\
Interface $^{e}$ & 5 \\
Binding $^{f}$ & 4 \\
Clash $^{g}$ & 6 \\
Voids $^{h}$ & 4 \\
Glycine $^{i}$ & 1 \\
${ }^{a}$ Disrupts a hydrogen bond. $^{b}$ Introduces a hydrophilic residue into the \\
protein core. $^{c}$ Introduces a hydrophobic residue on the surface. \\
${ }^{d}{ }$ Introduces or removes a charged residue from the protein core. \\
${ }^{e}$ Occurs at an interface according to difference in solvent accessibility. $^{f}$. ${ }^{f}$ Shows a specific binding interaction (hydrogen bond or van der Waals \\
contact) at an interface. ${ }^{g}$ Replacement sidechain clashes with its \\
surroundings. ${ }^{h}$ Introduces a void into the protein core. ${ }^{i}$ Parent resi- \\
due was a glycine with a backbone conformation not favoured for other \\
residues.
\end{tabular}

of hydrophilic residues into the core are the major destabilising factors in the GM1 Aio. Of the 44 structure-affecting amino acid differences between the GM1 and the A. faecalis Aio, eight amino acids are unique to GM1 that appear to be conserved, or are synonymous, in sequences of $A$. faecalis, NT-26 (a mesophilic alphaproteobacterium with a similar Aio temperature-activity profile to A. faecalis; $)^{33}$ and the thermophilic arsenite oxidiser Thermus thermophilus str. HB8. ${ }^{34}$ Of these eight amino acids, all in AioA, five introduce hydrophilic residues into the core of the structure: C32: Val-Thr; C231: Ile-Thr; C312: Gly-Thr; C469: LeuHis; C664: Gly-Asn; one disrupts a H-bond C668: Asp-Lys; one causes a clash: C779: Gly-Ala and one removes a buried charge: C713: Asp-Asn (Residue changes are written in the following format: 'PDB number: A. faecalis residue-GM1 residue'). The main mechanism for cold-adaptation of the GM1 Aio seems to be the insertion of hydrophilic residues into the hydrophobic core, which leads to destabilization of the enzyme structure, resulting in its increased activity at lower temperatures.

Our results are in agreement with the study of another member of the DMSO reductase family of molybdoenzymes, the cold-adapted periplasmic nitrate reductase (Nap) from Shewanella gelidimarina. ${ }^{35}$ Cold-adaptation in the S. gelidimarina Nap also resulted from destabilising changes to the structure and not changes to the active site or the presence of different cofactors.

\section{Experimental}

\section{GM1 growth conditions}

GM1 was grown in a Minimal Salts Medium (MSM) containing $0.04 \%$ yeast extract in the absence or presence of $5 \mathrm{mM}$ arsenite as described previously. ${ }^{18}$ All incubations were performed at $10{ }^{\circ} \mathrm{C}$ unless stated otherwise. Arsenic analyses were determined as described previously. ${ }^{18}$ The GM1-A strain, in which aioA was mutated, was grown in the presence of $5 \mu \mathrm{g} \mathrm{ml}^{-1}$ kanamycin. For the preparation of cell extracts and the purification of the Aio, GM1 was grown in the absence of arsenite in $2 \mathrm{~L}$ batch cultures (in $5 \mathrm{~L}$ flasks) until late-log phase $\left(72 \mathrm{~h}\right.$; $\mathrm{OD}_{600}$ : $0.15-0.17)$.

\section{Mutagenesis of aioA}

The GM1 aioA gene was disrupted by targeted gene disruption as has been done previously. ${ }^{15,25}$ Briefly, a $\sim 0.5 \mathrm{~kb}$ portion of the aioA gene ${ }^{18}$ was cloned into the suicide plasmid pJP5603 $\left(\mathrm{Km}^{\mathrm{R}}\right) .{ }^{36}$ The recombinant plasmid was transformed into Escherichia coli str. S17-1 $\lambda$ pir $^{37}$ and transferred into a spontaneous rifampicinresistant mutant of GM1 (GM1-R) by filter mating at $20^{\circ} \mathrm{C}$ for $24 \mathrm{~h}$ on a Luria Bertani (LB) agar plate. The conjugation mixture was plated on MSM containing $1.5 \%$ purified agar (Oxoid), $0.04 \%$ yeast extract (Oxoid), $100 \mu \mathrm{g} \mathrm{ml}{ }^{-1}$ rifampicin and $5 \mu \mathrm{g} \mathrm{ml} \mathrm{m}^{-1}$ kanamycin. The transconjugants were purified and selected for their ability to oxidise $5 \mathrm{mM}$ arsenite in MSM with $0.04 \%$ yeast extract as described previously. ${ }^{18}$

\section{Cloning and sequencing of the aio gene cluster}

The aio gene cluster was cloned by a 'plasmid rescue' technique. Genomic DNA from GM1-A was isolated using the Wizard ${ }^{\mathbb{R}}$ genomic DNA purification kit (Promega) and digested with the restriction enzyme ClaI. The linear fragments were circularised with T4 DNA ligase (Promega), transformed into E. coli str. JM109 $\lambda$ pir by electroporation and transformants containing pJP5603 and flanking GM1 genomic DNA were selected on LB containing $20 \mu \mathrm{g} \mathrm{ml}{ }^{-1} \mathrm{Km}$. Both strands of the aio gene cluster were sequenced by primer-walking. Sequencing was performed at the Wolfson Institute for Biomedical Research (WIBR) by capillary electrophoresis using a 3730XL Genetic Analyser with BigDye 3.1 (Applied Biosystems). Databases were searched using the BLAST suite (http://blast.ncbi.nlm.nih.gov/Blast.cgi) and open reading frames (ORFs) allocated using ORF Finder (http://www.ncbi.nlm. nih.gov/projects/gorf/). Signal peptide motifs were identified using SignalP $^{38}$ and TatP. $^{39}$ The GenBank accession number for the sequence of the GM1 aio gene cluster is EU106602.

\section{Preparation of GM1 cell fractions}

Total cell extracts were prepared as described previously ${ }^{6}$ using the previously determined optimum buffer of the Aio with the artificial electron acceptor 2,6-dichlorophenolindophenol (DCPIP), $50 \mathrm{mM}$ MES (pH 5.5). ${ }^{18}$ For the preparation of spheroplasts, cells were harvested and washed and spheroplasts prepared as described previously ${ }^{40}$ with the following modifications: (1) the harvested cells were suspended in ice-cold $750 \mathrm{mM}$ sucrose, $30 \mathrm{mM}$ Tris $/ \mathrm{HCl}(\mathrm{pH} 8)$ at a ratio of $0.5 \mathrm{~g}$ wet cells per $\mathrm{ml}$, (2) lysozyme was added at a concentration of $10 \mathrm{mg} \mathrm{g}^{-1}$ wet cells, (3) cells were incubated with lysozyme at room temperature for $1 \mathrm{~h}$, (4) after the addition of $15 \mathrm{mM}$ EDTA $\left(\mathrm{pH} 8,0^{\circ} \mathrm{C}\right)$, the suspension was incubated at $0{ }^{\circ} \mathrm{C}$ for $30 \mathrm{~min}$.

\section{Purification of the GM1 Aio}

The GM1 Aio was purified from the periplasm after spheroplast preparation. The periplasm was subjected to two ammonium sulphate precipitation steps, $50 \%$ and $75 \%$ saturation $\left(0{ }^{\circ} \mathrm{C}\right)$. The protein pellet was suspended in $50 \mathrm{mM}$ MES (pH 5.5) and desalted using a PD-10 desalting column (GE Healthcare) 
according to the manufacturer's instructions, with $50 \mathrm{mM}$ MES (pH 5.5) as the elution buffer. The sample $(3.5 \mathrm{ml})$ was passed through a $0.22 \mu \mathrm{m}$ filter (Millipore) and concentrated using a Vivaspin 20 device (10 000 MWCO) (Sartorius Stedium Biotech) to $<500 \mu \mathrm{l}$. The sample was applied to a SP sepharose fast flow HiPrep 16/10 cation exchange column (GE Healthcare) which had been pre-equilibrated with $50 \mathrm{mM}$ MES (pH 5.5). Chromatography was carried out at room temperature $\left(20-25{ }^{\circ} \mathrm{C}\right)$ at a flow rate of $5 \mathrm{ml} \mathrm{min}{ }^{-1}$. The Aio was eluted using a $1 \mathrm{M} \mathrm{NaCl}$ gradient in $50 \mathrm{mM}$ MES (pH 5.5). The $\mathrm{NaCl}$ gradient was as follows: (1) $0 \mathrm{M}$ for 2.25 column volumes and (2) a linear increase to $0.6 \mathrm{M}$ over 12 column volumes. Fractions containing Aio activity were pooled and concentrated. The sample was then applied to a Superdex 200 10/300 gel filtration column (GE Healthcare) pre-equilibrated with $50 \mathrm{mM}$ MES, $150 \mathrm{mM} \mathrm{NaCl}$ (pH 5.5). Chromatography was carried

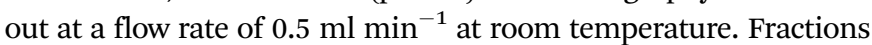
containing Aio activity were pooled and de-salted using a PD-10 desalting column.

\section{Heterologous expression and purification of the Alcaligenes faecalis Aio}

The $a i o B$ and aioA (aioBA) genes from $A$. faecalis were amplified without the native twin-arginine translocation (Tat) leader sequence using the following primers: Forward; 5'-GCTCTAGACGTACAACATTGCAGTACCCT- $3^{\prime}$ and Reverse: 5'-CGCTCGAGTCAGGCGAAGCGGCGGCTTTT- $3^{\prime}$. The restriction enzymes XbaI and XhoI (underlined above) were used for cloning into the expression vector, pPROEX-HTb in frame with the N-terminal histidine tag. The PCR reaction was performed for 35 cycles using the KOD proofreading DNA polymerase (Applied Biosystems) with a $55{ }^{\circ} \mathrm{C}$ annealing temperature as per the manufacturer's instructions.

The E. coli JM109 $\lambda$ pir strain was used for Aio expression. Expression of the Aio involved growing E. coli aerobically at $21{ }^{\circ} \mathrm{C}$ for $24 \mathrm{~h}$ in LB containing $100 \mu \mathrm{g} \mathrm{ml} \mathrm{m}^{-1} \mathrm{Ap}, 40 \mu \mathrm{M}$ IPTG (isopropyl $\beta$-D-1-thiogalactopyranoside) and $1 \mathrm{mM} \mathrm{Na} \mathrm{MoO}_{4}$.

Cells were harvested by centrifugation at $5464 \times g$ for $10 \mathrm{~min}$ $\left(4{ }^{\circ} \mathrm{C}\right)$ and the pellets washed by suspending them in binding buffer $\left(20 \mathrm{mM} \mathrm{KH} \mathrm{KH}_{4}, 500 \mathrm{mM} \mathrm{NaCl}, 20 \mathrm{mM}\right.$ imidazole, $\mathrm{pH}$ 7.3). The cells were centrifuged at $15180 \times g$ for $10 \mathrm{~min}$ $\left(4{ }^{\circ} \mathrm{C}\right)$ and the pellet resuspended in binding buffer $\left(10 \mathrm{ml} \mathrm{g}^{-1}\right.$ wet weight cells). Cells were disrupted by two passages through a French press (13000 psi) and the cell debris removed by centrifugation at $30000 \times g\left(4{ }^{\circ} \mathrm{C}\right)$. The supernatant was filtered through a $0.22 \mu \mathrm{M}$ filter (Millipore) and loaded onto a HisTrap-Ni Sepharose High Performance column (GE Healthcare) that had been pre-equilibrated with binding buffer. Chromatography was carried out at $1 \mathrm{ml} \mathrm{min}{ }^{-1}$ at room temperature and unbound proteins removed with 30 column volumes of binding buffer followed by a gradient of 30 column volumes with elution buffer (20 mM KH $\mathrm{KH}_{4} 500 \mathrm{mM} \mathrm{NaCl}, 500 \mathrm{mM}$ imidazole, $\mathrm{pH}$ 7.3). Fractions that contained Aio activity (Aio was eluted at $295 \mathrm{mM}$ imidazole) were pooled and the sample was concentrated using a Vivaspin 20 (MWCO 50 000) (Satorius Stedium Biotech) centrifugal concentrator and loaded on to a Superdex 200 10/300 gel filtration column (GE Healthcare) that had been pre-equilibrated with $50 \mathrm{mM}$ MES, $150 \mathrm{mM} \mathrm{NaCl}$ (pH 6.0). Gel filtration chromatography was carried out at a flow rate of $0.5 \mathrm{ml} \mathrm{min}{ }^{-1}$ at room temperature. Fractions containing Aio activity were pooled and the buffer exchanged using a PD-10 desalting column (GE Healthcare), according to the manufacturer's instructions, with $50 \mathrm{mM}$ MES (pH 6.0) serving as the elution buffer. The sample was concentrated using a Vivaspin 20 (MWCO 50 000) (Satorius Stedium Biotech) and Micron YM-30 (Millipore) centrifugal concentrators and enzyme assays performed immediately.

\section{Enzyme assays and protein determination}

Aio and malate dehydrogenase enzyme assays were done as described previously. ${ }^{6}$ The results of the kinetics are from two independent experiments with three replicates for each arsenite concentration tested, so there are a total of 6 values for each concentration. All assays were performed at $24{ }^{\circ} \mathrm{C}$ unless otherwise stated. The temperature of the enzyme assays was controlled with a Varian Cary dual cell Peltier. Reaction mixtures were incubated at the cell temperature for 5 minutes prior to the addition of arsenite.

Protein concentrations were determined using the Bradford method $^{41}$ and BioRad reagent according to the manufacturer's instructions and bovine serum albumin served as the standard.

The oxidised and reduced spectra of the Rhizobium sp, str. NT-26 cytochrome $c_{552},{ }^{15}$ horse heart cytochrome $c$ (Sigma), and $P$. aeruginosa azurin (Sigma) were recorded as described previously ${ }^{15,26}$ with $20 \mu \mathrm{g}$ of electron acceptor and $3 \mu \mathrm{g}$ of Aio.

\section{Subunit composition and cofactor analysis}

SDS-PAGE of denatured protein was performed as described previously ${ }^{40}$ with low molecular weight standards (GE Healthcare). For cofactor analysis purified Aio was prepared as described previously $^{25}$ and analysed for Fe, Co, Ni, Cu, Zn, Mo and W, by inductively coupled plasma-mass spectrometer (ICP-MS) (Varian) at the Mineralogy Department, Natural History Museum, London, UK.

\section{Homology modelling}

Modelling of the GM1 Aio was performed with Modeller ${ }^{42}$ using the $A$. faecalis Aio crystal structure as a template (PDB code $1 \mathrm{~g} 8 \mathrm{j}$, chains $\mathrm{C}$ and D). ${ }^{12}$ The alignment was hand-modified to accommodate the insertion at C505-C506 and deletion at C651 to minimise disruption of the parent structure. Structural effects of amino acid differences were analysed using the SAAPdap pipeline of the SAAPdb database ${ }^{32}$ treating each difference as a single mutation in the A. faecalis structure.

\section{Conclusions}

GM1 is a psychrotolerant arsenite oxidiser that possesses an Aio that is adapted to function at cold temperatures. Compared to its mesophilic counterparts the GM1 Aio has the same subunit composition and contains the same cofactors but it has a much higher reaction rate and a lower affinity for arsenite. Coldadaptation of GM1 Aio is probably caused by an increase in the enzyme's flexibility which appears to result from changes in the overall protein structure and not by alterations specific to 
the active site or cofactors. The insertion of hydrophilic residues into the core is a major destabilising factor in the GM1 Aio. The aerobic arsenite oxidase is an ancient bioenergetic enzyme ${ }^{27,43,44}$ and understanding the reaction mechanism from psychrophilic, mesophilic and thermophilic homologues will not only improve our understanding of its evolution but should also aid our development of a broad-temperature range arsenite biosensor.

\section{Acknowledgements}

THO was supported by a Natural Environment Research Council PhD studentship (14404A). MDH is supported by a Medical Research Council PhD studentship (G0800112). We would like to thank Barbara Schoepp-Cothenet and Gretchen Anderson for supplying the A. faecalis strain and helpful discussions. We would also like to thank Stanislav Strekopytov for performing the metal analyses of the arsenite oxidase.

\section{References}

1 J. O. Nriagu, in Environmental chemistry of arsenic, ed. W. T. Frankenberger, Marcel Dekker, New York, 2002, ch. 1, pp. 1-26.

2 P. L. Smedley and D. G. Kinniburgh, Appl. Geochem., 2002, 17, 517.

3 M. F. Hughes, Toxicol. Lett., 2002, 133, 1.

4 P. Ravenscroft, H. Brammer and K. Richards, Arsenic pollution a global synthesis, Wiley-Blackwell, UK, 2009.

5 D. K. Nordstrom, Science, 2002, 296, 2143.

6 J. M. Santini, L. I. Sly, R. D. Schnagl and J. M. Macy, Appl. Environ. Microbiol., 2000, 66, 92.

7 R. S. Oremland and J. F. Stolz, Science, 2003, 300, 939.

8 H. H. Green, S. Afr. J. Sci., 1918, 14, 465.

9 T. H. Osborne and J. M. Santini, in The Metabolism of Arsenite, ed. J. M. Santini and S. A. Ward, CRC Press, Boca Raton, Florida, 2012, ch. 5, pp. 61-69.

10 M. C. Lett, D. Muller, D. Lièvremont, S. Silver and J. M. Santini, J. Bacteriol., 2012, 194, 207.

11 A. G. McEwan, J. P. Ridge, C. A. McDevitt and P. Hugenholtz, Geomicrobiol. J., 2002, 9, 3.

12 P. J. Ellis, T. Conrads, R. Hille and P. Kuhn, Structure, 2001, 9, 125.

13 M. D. Heath, B. Schoepp-Cothenet, T. H. Osborne and J. M. Santini, in The Metabolism of Arsenite, ed. J. M. Santini and S. A. Ward, CRC Press, Boca Raton, Florida, 2012, ch. 7, pp. 81-97.

14 A. Lieutaud, R. van Lis, S. Duval, L. Capowiez, D. Muller, R. Lebrun, S. Lignon, M. L. Fardue, M. C. Lett, W. Nitschke and B. Schoepp-Cothenet, J. Biol. Chem., 2010, 285, 20442.

15 J. M. Santini, U. Kappler, S. A. Ward, M. J. Honeychurch, R. N. vanden Hoven and P. V. Bernhardt, Biochim. Biophys. Acta, 2007, 1767, 189.

16 S. Sardiwal, J. M. Santini, T. H. Osborne and S. Djordjevic, FEMS Microbiol. Lett., 2010, 313, 20.

17 G. Liu, M. Liu, E. H. Kim, W. S. Maaty, B. Bothner, B. Lei, C. Rensing, G. Wang and T. R. McDermott, Environ. Microbiol., 2012, 14, 1624.
18 T. H. Osborne, H. E. Jamieson, K. A. Hudson-Edwards, D. K. Nordstrom, S. R. Walker, S. A. Ward and J. M. Santini, BMC Microbiol., 2010, 10, 205.

19 Y. Huang, H. Li, C. Rensing, K. Zhao, L. Johnstone and G. Wang, J. Bacteriol., 2012, 194, 1635.

20 S. Koechler, J. Cleiss-Arnold, C. Proux, O. Sismeiro, M. A. Dillies, F. Goulhen-Chollet, F. Hommais, D. Lièvremont, F. ArsènePloetze, J. Y. Coppée and P. N. Bertin, BMC Microbiol., 2010, 10, 53.

21 D. R. Kashyap, L. M. Botero, W. L. Franck, D. J. Hassett and T. R. McDermott, J. Bacteriol., 2006, 188, 1081.

22 N. R. Stanley, T. Palmer and B. C. Berks, J. Biol. Chem., 2000, 275, 11591.

23 G. L. Anderson, J. Williams and R. Hille, J. Biol. Chem., 1992, 267, 23674.

24 K. S. Prasad, V. Subramanian and J. Paul, Biometals, 2009, 22, 711.

25 J. M. Santini and R. N. vanden Hoven, J. Bacteriol., 2004, 186, 1614.

26 R. N. vanden Hoven and J. M. Santini, Biochim. Biophys. Acta, 2004, 1656, 148.

27 S. Duval, A. L. Ducluzeau, W. Nitschke and B. SchoeppCothenet, BMC Evol. Biol., 2008, 8, 206.

28 G. Feller and C. Gerday, Nat. Rev. Microbiol., 2003, 1, 200.

29 K. S. Siddiqui and R. Cavicchioli, Annu. Rev. Biochem., 2006, 75, 403.

30 D. Georlette, B. Damien, V. Blaise, E. Depiereux, V. N. Uversky, C. Gerday and G. Feller, J. Biol. Chem., 2003, 278, 37015.

31 S. D'amico, J. C. Marx, C. Gerday and G. Feller, J. Biol. Chem., 2003, 278, 7891.

32 J. M. Hurst, L. E. M. McMillan, C. T. Porter, J. Allen, A. Fakorede and A. C. R. Martin, Hum. Mutat., 2009, 30, 616. 33 T. P. Warelow and J. M. Santini, Unpublished work.

34 T. M. Gihring, G. K. Druschel, R. B. McClesky, R. J. Hamers and J. F. Banfield, Environ. Sci. Technol., 2001, 19, 3857.

35 P. J. Simpson and R. Codd, Biochem. Biophys. Res. Commun., 2011, 414, 783.

36 R. J. Penfold and J. M. Pemberton, Gene, 1992, 118, 145.

37 R. Simon, U. Priefer and A. Pühler, Biotechnology, 1983, 1, 784.

38 T. N. Petersen, S. Brunak, G. von Heijne and H. Nielsen, Nat. Methods, 2011, 8, 785.

39 D. Bendtsen, H. Nielsen, D. Widdick, T. Palmer and S. Brunak, BMC Bioinf., 2005, 6, 167.

40 T. Krafft and J. M. Macy, Eur. J. Biochem., 1998, 255, 647.

41 M. M. Bradford, Anal. Biochem., 1976, 72, 248.

42 N. Eswar, M. A. Marti-Renom, B. Webb, M. S. Madhusudhan, D. Eramian, M. Shen, U. Pieper and A. Sali, Curr. Protoc. Bioinformatics, 2006, Suppl. 15, 5.6.1.

43 R. van Lis, W. Nitschke, S. Duval and B. Schoepp-Cothenet, in The Metabolism of Arsenite, ed. J. M. Santini and S. A. Ward, CRC Press, Boca Raton, Florida, 2012, ch. 10, pp. 125-144.

44 E. Lebrun, M. Brugna, F. Baymann, D. Muller, D. Lièvremont, M.-C. Lett and W. Nitschke, Mol. Biol. Evol., 2003, 20, 686. 